\title{
BMJ Open Association between progression-free survival and health-related quality of life in oncology: a systematic review protocol
}

\author{
Bruno Kovic, ${ }^{1}$ Gordon Guyatt, ${ }^{1,2}$ Michael Brundage, ${ }^{3}$ Lehana Thabane, ${ }^{1}$ \\ Neera Bhatnagar, ${ }^{4}$ Feng Xie ${ }^{1,5}$
}

To cite: Kovic B, Guyatt G, Brundage $\mathrm{M}$, et al. Association between progression-free survival and health-related quality of life in oncology: a systematic review protocol. BMJ Open 2016;6: e012909. doi:10.1136/ bmjopen-2016-012909

- Prepublication history and additional material are available. To view please visit the journal (http://dx.doi.org/ 10.1136/bmjopen-2016012909).

Received 31 May 2016 Revised 9 August 2016 Accepted 12 August 2016

CrossMark

For numbered affiliations see end of article.

Correspondence to

Dr Feng Xie;

fengxie@mcmaster.ca

\section{ABSTRACT}

Introduction: There is an increasing number of new oncology drugs being studied, approved and put into clinical practice based on improvement in progression-free survival, when no overall survival benefits exist. In oncology, the association between progression-free survival and health-related quality of life is currently unknown, despite its importance for patients with cancer, and the unverified assumption that longer progression-free survival indicates improved health-related quality of life. Thus far, only 1 study has investigated this association, providing insufficient evidence and inconclusive results. The objective of this study protocol is to provide increased transparency in supporting a systematic summary of the evidence bearing on this association in oncology.

Methods and analysis: Using the OVID platform in MEDLINE, Embase and Cochrane databases, we will conduct a systematic review of randomised controlled human trials addressing oncology issues published starting in 2000. A team of reviewers will, in pairs, independently screen and abstract data using standardised, pilot-tested forms. We will employ numerical integration to calculate mean incremental area under the curve between treatment groups in studies for health-related quality of life, along with total related error estimates, and a $95 \%$ $\mathrm{Cl}$ around incremental area. To describe the progression-free survival to health-related quality of life association, we will construct a scatterplot for incremental health-related quality of life versus incremental progression-free survival. To estimate the association, we will use a weighted simple regression approach, comparing mean incremental health-related quality of life with either median incremental progression-free survival time or the progression-free survival $H R$, in the absence of overall survival benefit.

Discussion: Identifying direction and magnitude of association between progression-free survival and health-related quality of life is critically important in interpreting results of oncology trials. Systematic evidence produced from our study will contribute to improvement of patient care and practice of evidence-based medicine in oncology.

\section{INTRODUCTION}

Cancer is a devastating disease, causing high morbidity and mortality. Over time, a variety of anticancer treatments have emerged to deal with the many different forms of cancer, all having the same guiding principle-they should only be considered for use when they provide important benefit (ie, improved quantity and/or quality of patient survival). ${ }^{12}$ Unfortunately, it is not currently always known whether treatment does provide important benefit to patients, which is critical to clinical decision-making. ${ }^{3}$

In oncology, overall survival (OS) is an objective end point since it represents survival time, a direct and unambiguous patient benefit. OS has long been regarded by the oncology community at large, as well as drug regulatory bodies such as the U.S. Food and Drug Administration (FDA), as the gold standard for demonstrating clinical benefit. ${ }^{4}$ Using OS as an end point has its limitations, in particular the need for larger sample sizes and longer follow-up. ${ }^{2} 4$ The other wellrecognised primary consideration in cancer therapy is the improvement of health-related quality of life (HRQoL), ${ }^{1}{ }^{4}$ which in oncology generally refers to symptomatic improvement. HRQoL is a patient-reported outcome that reflects the subjective feeling of patients about their own health. HRQoL has been recognised as an important outcome measure to patients, since basing decisions on only survival ignores other vital dimensions of great concern to patients. Its importance is highlighted by the American Society of Clinical Oncology recommendations for patients, which identifies research into the maximisation of HRQoL as a research priority in oncology. ${ }^{1}$

Although important clinical benefit should ultimately be defined in oncology by prolonged survival/improved HRQOL, 
treatments are also accepted by regulatory authorities on the basis of established surrogates. ${ }^{4}$ One currently accepted surrogate end point being used is progressionfree survival (PFS), defined as the time from randomisation in a clinical trial to objective tumour progression or death. Sometimes time to progression (TTP) is used interchangeably with PFS. ${ }^{5}$

Cancer disease progression is typically assessed via medical imaging at scheduled intervals, and determined based on one of the four changes: appearance of one or more new lesions, increase in size of target measurable lesions, clear increase in non-target disease and worsening of symptoms of disease. ${ }^{5}$ Progression is an outcome that was originally developed as a measurement tool to describe change in tumour burden during therapy; thus, it was intended for use in phase II screening trials of new drugs, and not used to denote clinical benefit. Essentially this use was focused on identifying signals of activity in early drug development, and not rooted in benefit for patients. $^{2}$

In spite of the first use of PFS as an indicator of treatment effect on tumours, PFS use as an important end point in phase III randomised controlled trials (RCTs) has increased over time, with this being reflected in RCT publications and drug regulatory approvals. For example, the proportion of breast, colorectal and lung cancer RCT publications using PFS/TTP as the primary end point in the Journal of Clinical Oncology went from 0\% during 1975-1984 to 26\% during 2005-2009, and the approval rates of drug indications based on PFS/ TTP end points, as reported in different studies, were found to be $23 \%$ during $2005-2007$ vs $29 \%$ during 2000-2010. ${ }^{2}$

Increasing the use of PFS as the outcome measure of choice in oncology trials stems from various sources. First, in contrast to the limitations of the gold standard OS, PFS studies can be shorter and have fewer patients, providing results faster and at less expense. ${ }^{5}$ Second, new drugs are now being targeted towards cytostatic rather than cytotoxic molecular mechanisms of action, which makes using PFS to measure cytostatic effects on tumours a logical choice. ${ }^{6}$

There are also major disadvantages to using PFS as a primary outcome, reflecting the uncertainty of the importance of the outcome to patients. If prolonged PFS was associated with prolonged OS, it would clearly be important. However, since prolonged PFS is not necessarily associated with prolonged OS, the only reason it would be of importance to patients is if it were associated with improved HRQoL, which is by no means certain. For instance, any HRQoL benefit of PFS may be eliminated or even reversed by HRQoL impairment as a result of adverse events (AEs) of the treatment required to achieve prolonged PFS. ${ }^{5}$

This fundamental problem with PFS is reflected by the various recent publications from oncological experts who are concerned with the validity of using PFS as a primary outcome for the evaluation of new treatments. ${ }^{125}$ As Booth highlights, the growing use of PFS as a primary end point is not based on its surrogacy for either OS/HRQoL, but on the conveniences of shorter and faster trials and evaluations, offering little benefit to patients when this is the basis for drug approvals, since there should be good evidence for PFS as a surrogate for OS/HRQoL. ${ }^{2}$

A surrogate end point is defined as a measure validated as an adequate substitute for an outcome of intrinsic value to patients: how a patient feels, functions or survives. Treatment effect observed on a valid surrogate end point should reliably and precisely predict treatment effect on the outcome being replaced. ${ }^{6}$ In terms of PFS being a proper surrogate for how a patient survives (ie, OS), data suggest that PFS is only a valid surrogate for OS in colorectal cancer, and certain types of ovarian cancer, with data for other types of cancer such as breast, prostate and lung cancer not supporting the surrogate relationship. ${ }^{2}{ }^{6}$ Indeed, although there is some evidence for the surrogacy of PFS for OS, correlation between these is variable and unpredictable. PFS failing surrogacy for OS, however, may not be a problem if it is a valid surrogate for HRQoL (ie, how a patient feels/functions).

Unfortunately, there is a paucity of data examining the surrogacy of PFS for HRQoL; however, many drugs are approved based on PFS benefit in the absence of OS benefit. The question which is therefore raised is what benefits these approved drugs can bring to patients if PFS does not represent a good surrogate for HRQoL? There are insufficient studies directly measuring the value and benefits of PFS for patients. Many novel cancer treatment RCTs report outcomes in PFS and few trials collect good HRQoL data, even though HRQoL is important to patient care, since outcomes may not provide benefits to patients experiencing AEs. ${ }^{5}$ An example of this scenario occurred with bevacizumab, which was initially approved for breast cancer by the FDA based on 5.9 months of PFS gain, with no accompanying OS or HRQoL gain and many associated AEs. Further trials of this treatment revealed smaller PFS gains and more associated AEs than originally found, and ultimately lead the FDA to revoke their approval for this indication. ${ }^{5}$

Advocates for PFS claim that delaying progression delivers patient-important benefit since being progressionfree results in stability of disease and symptoms that leads to a reduction in physical and psychological morbidity, and thus improves HRQoL. However, as Fallowfield noted, the HRQoL benefits of increased PFS must be balanced against the toxicity of drug therapy. ${ }^{5}$ The limitations in measurement of HRQoL outcomes often leave the matter of net benefits of increased PFS versus increased treatment toxicity uncertain. ${ }^{5}$

Until now there has only been one study, a report published by the Agency for Healthcare Research and Quality (AHRQ), ${ }^{6}$ that has attempted to gather the evidence and perform a systematic analysis of the 
association between PFS and HRQoL. The AHRQ report examined the association of PFS with HRQoL and related outcomes, such as disease symptoms, for agents where PFS was the primary outcome used to assess treatment benefit. Unfortunately, only four studies proved eligible for the AHRQ analysis, and the quality of the evidence was deemed insufficient to make any conclusion about the PFS-HRQoL association.

In evaluating the PFS-HRQoL association, the AHRQ report had some important limitations. The search strategy used in the AHRQ report was not sufficiently comprehensive. First, their search was limited to only the specific set of oncology drugs that were approved based on PFS, ignoring all other studies containing PFS and HRQoL data. Second, they limited their search to the 2004-2012 timeframe, a timeframe consistent with drugs receiving approval for a primary outcome of PFS, but again ignoring a few years of data where PFS was reported in RCTs. Finally, and most importantly, they only chose studies that included a direct quantitative statistical comparison of PFS to HRQoL, limiting their available data given that it is very rare that a direct comparison between PFS and HRQoL is made in published studies.

If a broader search with fewer limits to capture more of the available PFS and HRQoL data was performed, more studies would be found. Greater available studies would allow for a quantitative analysis, including sensitivity and subgroup analyses, which would lead to more robust conclusions on the PFS-HRQoL association.

Given the increased use of PFS as the primary outcome of importance in oncology trials of new drugs, the importance of HRQoL to patients in oncology treatments and trials, and the lack of a consistent surrogate relationship between PFS and OS, it is important to all stakeholders, including patients, clinicians, payers and all decision makers, to identify the currently unknown PFS-HRQoL association. Without this evidence, it remains possible that patients are receiving toxic and expensive treatments on the basis of PFS prolongation, but are not experiencing any benefit of value to them in the form of prolonged survival or HRQoL.

In order to address this important gap in the current oncology literature, we will perform a systematic review and quantitative analyses to determine the PFS-HRQoL association in studies of oral or intravenous or intraperitoneal or intrapleural chemotherapy and biological therapy designed to improve disease-related outcomes among patients with cancer in a RCT setting.

\section{METHODS AND ANALYSIS}

\section{Study overview}

We will conduct a systematic review of RCTs using the standard methodology as described by the Cochrane Collaboration. ${ }^{7}$ Our protocol adheres to the Preferred Reporting Items for Systematic reviews and Meta-Analyses for Protocols 2015 (PRISMA-P 2015) 9 guidelines. Additionally, in order to increase the availability and accessibility of the a priori methods of our systematic review, prior to the initiation of data abstraction, we plan on registering our study protocol on the PROSPERO (International Prospective Register of Ongoing Systematic Reviews) ${ }^{10}{ }^{11}$ international register.

\section{Literature search}

With the help of an experienced research librarian, using the OVID platform, we will design and run searches in MEDLINE, Embase and Wiley's Cochrane Library (ie, Cochrane Central Register of Controlled Trials-CENTRAL) databases using medical subject headings (MeSH) and text words. The MEDLINE search will allow for the capture of RCTs published in the core clinical journals, or Abridged Index Medicus, as defined by the National Library of Medicine. ${ }^{12}$ References of retrieved publications will be scanned to identify any other relevant publications. In order to increase the comprehensiveness of our review, our search will have no language limitations.

Search strategies will be built that combine terms from the three key areas of the disease, HRQoL and cancer treatment, with 'AND' modifiers. For the terms in the disease key area, the five major categories of cancer will be used, as identified by major cancer organisations such as the National Cancer Institute (NCI) and others. ${ }^{13} 14$ The treatment key area terms will be constructed around chemotherapy and biological-type cancer therapies acting as primary agents in cancer treatment. Chemotherapy and biological therapies identified in the search will be defined as stated by the NCI. ${ }^{15} 16$ To be even more comprehensive, in our treatment key area terms, we will include the list of specific drugs that have been approved by the FDA based only on PFS outcome for cancer as identified in the AHRQ report, ${ }^{6}$ since we know that these drugs are of interest to our research question. Additionally, to be able to form conclusions using higher quality studies that are the standard of approval for new interventions and therefore our context of interest, the focus will be only on RCTs. Therefore, a fourth key area with an AND modifier will be used in the form of an RCT filter in order to focus the search results further. The MEDLINE search has been constructed first and will be used as the baseline search strategy on which to construct the Embase and Cochrane searches. The MEDLINE strategy can be found in online supplementary appendix 1 .

The outcome of PFS was not included in the search strategy, and instead will be screened on in order to adequately capture HRQoL post hoc analysis publications corresponding to RCTs reporting PFS outcomes. HRQoL post hoc analysis publications might not mention PFS, and HRQoL data are frequently reported in these later types of publications, instead of at the time of publication on primary outcomes of efficacy and safety. Various key articles known to be eligible for data abstraction were identified a priori, with some of these 
being the final four from the AHRQ report, and these were used to perform a quality check on the MEDLINE search, to check for inclusion of these key articles in the search strategy.

\section{Eligibility criteria}

The following are our eligibility criteria.

Inclusion criteria:

1. The study is a RCT;

2. Only human participants;

3. The study is examining only cancer disease (ie, malignant neoplasm), with a focus on cancer treatment;

4. The study was published during 2000-2016 (deemed by expert opinion to include most RCTs using PFS as a primary outcome);

5. The study reported PFS outcome estimates for either all trial arms or the difference between arms, in terms of median PFS times or HR difference;

6. Report of HRQoL measures (generic, specific or utility);

7. Oral or intravenous or intraperitoneal or intrapleural chemotherapy/biologicals used as the primary agent in treating cancer, including: immunotherapy (cytokines and antibodies), gene therapy and targeted therapies (tyrosine kinase inhibitors, apoptosis-inducing drugs, treatment vaccines, antiangiogenesis inhibitors).

Exclusion criteria:

1. The presence of statistically significant OS benefits;

2. Median or HR PFS outcome measures not provided for all arms;

3. Fewer than two discrete HRQoL assessments/measurements provided in study (baseline and at least one follow-up assessment must be provided);

4. Error estimates, in the form of variances or SDs not reported for all HRQoL measurements;

5. All time durations between successive HRQoL measurements not reported;

6. All sample sizes at each HRQoL measurement point not reported;

7. All non-oral or non-intravenous or nonintraperitoneal or non-intrapleural chemotherapy/ biologicals such as surgical therapy or radiotherapy;

8. Any agents used against cancer that are used as supportive, secondary or preventive agents such as hormone therapy or preventive vaccines.

\section{Review process}

A team of screeners and abstractors will be recruited to assist with the review process. Teams of two trained reviewers will perform citation and full-text screening and data abstraction, in duplicate and independently, for citations distributed to each team at random. Each team will attempt to resolve all discrepancies by consensus through discussion with their respective team member, but if unsuccessful, adjudication to resolve the discrepancy will be performed by one of the two arbitrators (BK and FX). The arbitrator will independently review the study and respective discrepancy, before discussing their final decision with the team members.

We will use electronic forms developed with DistillerSR (Evidence Partners, Ottawa, Canada) and Microsoft Excel software for study screening and data abstraction, respectively. Level 1 title and abstract screening and level 2 full-text screening forms will be constructed using eligibility criteria. All forms will be pilot-tested before distribution and implementation in the review process. Before the review formally starts, we will conduct calibration exercises to ensure consistency across reviewers. These exercises will consist of project and role review meetings, as well as sample data review exercises. For the purposes of evaluating reproducibility, $\kappa$ will be calculated and reported for level 2 screening.

Since PFS is not being included in the search strategy, the presence of PFS outcome measures being reported for all arms in each study will be screened on during level 1 screening, if possible, in order to reduce the large amount of studies expected to not contain these required data.

Since the relationship between PFS and HRQoL is important in the absence of OS benefit, and HRQoL reporting would be expected to be less thorough when OS benefit is present, the focus will be on studies reporting no statistically significant OS benefit.

\section{Data abstraction}

We will extract information on our primary outcomes of PFS and HRQoL, regardless of statistically significant differences reported in studies. Median PFS times and/or PFS HRs will be extracted for every intervention and control group. Mean HRQoL point values for the overall physical domain base case will be extracted for every group at every time point in the study. Error estimates around all mean HRQoL point values and, if available, around PFS values will also be extracted. For the HRQoL measurements, time durations between each successive measurement in each group and the sample size at each measurement point will also be recorded.

To perform sensitivity and subgroup analyses, the following additional data from each study will also be recorded:

- HRQoL measurements for the overall global and overall emotional HRQoL domains;

- potential bias as per the Cochrane risk of bias instrument, ${ }^{17}$ with a focus on critical sources (ie, the presence of loss to follow-up (LTFU) issues defined as $>20 \%$ attrition or problem self-identified by study, failure to follow patients after progression and the absence of blinding);

- cancer types and/or stages;

- the presence or absence of industry funding.

\section{Data analysis}

Primary analysis

Our data analysis will be informed by observation of the independent point values of median incremental PFS or 
PFS HR, and the incremental HRQoL values for one intervention versus another (control) provided by each study. All individual HRQoL and PFS point value and error estimate measurements will be standardised across studies to ensure that comparisons for incremental areas and progression-free times are commensurate with each other.

To calculate incremental PFS, we will use either one of two measurements, depending on available data. The first method consists of taking the difference in median PFS time duration between arms as reported in the study, or the second method consists of using the PFS HR as reported in the study.

In order to determine the overall PFS-HRQoL association for the base case, a HRQoL domain must be selected that can summarise HRQoL effects across different cancer types and maintain the same types of comparisons across different instruments (since instruments can vary in the domains they capture). Therefore, for the base case scenario analysis of the association of PFS with HRQoL, we will use the overall physical HRQoL domain from each study.

Data analyses for incremental HRQoL will be performed by using an area under the curve (AUC) approach, following the trapezoidal rule, to calculate the treatment effect on HRQoL which requires at least two measures of HRQoL. Incremental AUC differences between intervention and control arm group curves for consecutive HRQoL measurements per group in each RCT, regardless of statistical significance, will be calculated for the entire timeframe duration of HRQoL measurements, up to the shortest HRQoL arm group duration measurement provided (no imputation assumptions will be made). The whole curve with the lower HRQoL baseline score will be adjusted upwards to correct for the imbalance at baseline HRQoL. Refer online supplementary appendix 2 for a more detailed description of the formulas and calculations that were developed for the data analysis.

To describe the PFS-HRQoL association (ie, whether or not an improvement in PFS corresponds with an improvement in HRQoL), we will construct a scatterplot, with the axes consisting of incremental HRQoL versus incremental PFS, and with each point on the plot representing the data extracted from each individual study. Through the visual observation of this plot, we may be able to notice/describe an overall trend of how one variable changes versus another. Figure 1 shows two possible scatterplot example scenarios.

To estimate the PFS-HRQoL association (ie, how much the dependent variable HRQoL varies with a corresponding increase in the independent variable PFS), we will use a weighted simple regression approach with an assumption of no error for the independent incremental PFS variable. This assumption aligns with the potential unavailability of error estimates for PFS, since to the best of our knowledge, it is not usually reported in studies. The regression will be performed by using the dependent incremental HRQoL area point estimate, versus the independent incremental median PFS time estimate or PFS HR for each study. We will run this simple regression using a regression formula (ie, $y=\alpha+\beta$ $(\mathrm{x})$, where $\mathrm{x}$ is measured without error). Finally, each study point in the regression analysis will be weighted by the inverse of the total variance (incremental area variance), to account for the influence of each study estimate on the regression line, originating from the uncertainty due to different sample sizes across studies.

\section{Sensitivity/subgroup analyses and a priori hypotheses}

Sensitivity and subgroup analyses will be performed on the base case scenario of the association of PFS with HRQoL. First, we will perform subgroup analyses for overall global and emotional HRQoL domains, and hypothesise a priori that the overall global HRQoL domain will have the same direction of association as the overall physical HRQoL domain, although the magnitude of the associations may vary due to other domains having lesser or greater impact on overall global HRQoL.

The AHRQ report identified the three most critical sources of potential bias, which are especially related to the association between PFS and HRQoL, these being: LTFU issues defined as $>20 \%$ attrition, failure to follow patients after progression and failure to blind. Studies will be evaluated for potential bias with the Cochrane
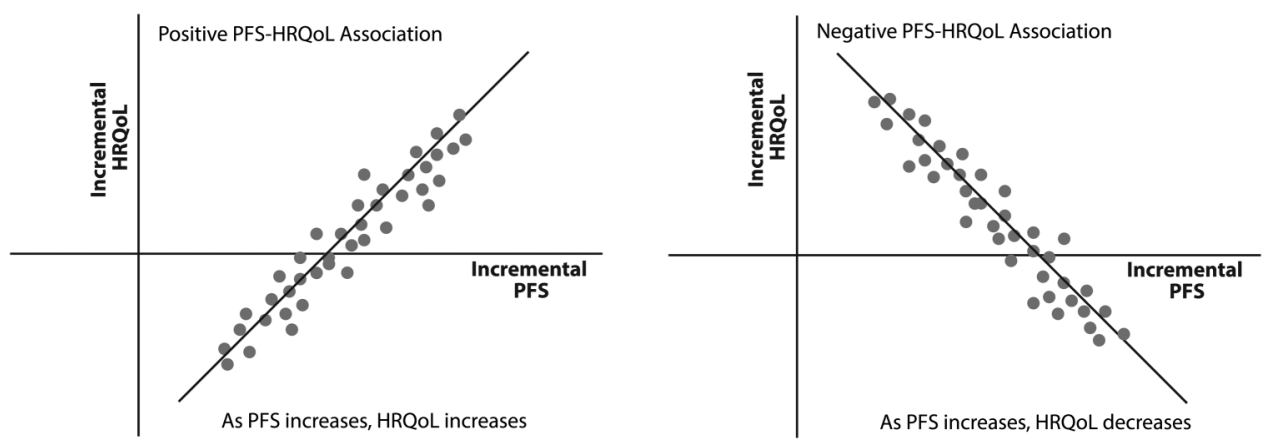

Figure 1 Scatterplots for incremental HRQoL (y-axis) versus incremental PFS (x-axis). HRQoL, health-related quality of life; PFS, progression-free survival. 
risk of bias instrument, ${ }^{17}$ with a focus on the three critical sources, and sensitivity analyses will be performed by excluding studies deemed to have high risk of bias on any of these critical variables. Our a priori hypothesis regarding study quality is that the inclusion of studies not measuring past median progression, or with significant LTFU/attrition problems $(>20 \%$ attrition), will decrease the positive PFS-HRQoL association, since incremental HRQoL benefits derived from increased PFS between arms are expected to be seen more the longer the follow-up, due to more time to get over adverse treatment effects and for the positive impact of staying free of recurrence to manifest itself.

Finally, to explore some other potential predictors for their potential effect on the PFS-HRQoL association, oncology-type subgroup analysis and a sensitivity analysis excluding industry funded RCTs may be performed, depending on available data. Table 1 presents a summary of the main methodological aspects of this study.

\section{DISCUSSION}

\section{Strengths and limitations}

Our study has several strengths. First, we will employ rigorous systematic review methodology, including welldesigned and comprehensive search strategies, screening that is guided by explicit eligibility criteria, and the use of standardised and pilot-tested screening and abstraction forms. Second, we will maximise reproducibility of our review methods by conducting calibration exercises throughout the review process to ensure consistency across reviewers, and we will report on the $\kappa$ statistic for level 2 screening to identify any potential problems with regards to reproducibility. Our comprehensive search will provide an informative estimate on the PFS-HRQoL association. Our sophisticated analytic strategy will optimally explore the association, and we will conduct sensitivity analyses to address the robustness of the results and a subgroup analysis to determine whether the association varies across specific domains of HRQoL.

We anticipate several limitations to our analysis. First, reported median PFS will not always match for time duration with HRQoL measurements provided, resulting in a time mismatch between these outcomes. The greater the difference in the proportion of patients in intervention and control groups without a recurrence, and the longer time in which patients with and without a recurrence live with their conditions, the greater the net HRQoL difference between the two groups is likely to be. The difference in the proportion of recurrence, as well as the time lived with or without a recurrence and, thus, the net difference in HRQoL in intervention and control groups will increase with the duration of follow-up. Thus, studies with briefer or truncated follow-up with respect to HRQoL, but longer follow-up with respect to PFS-and we have prior knowledge that such differences in follow-up will occur-are liable to

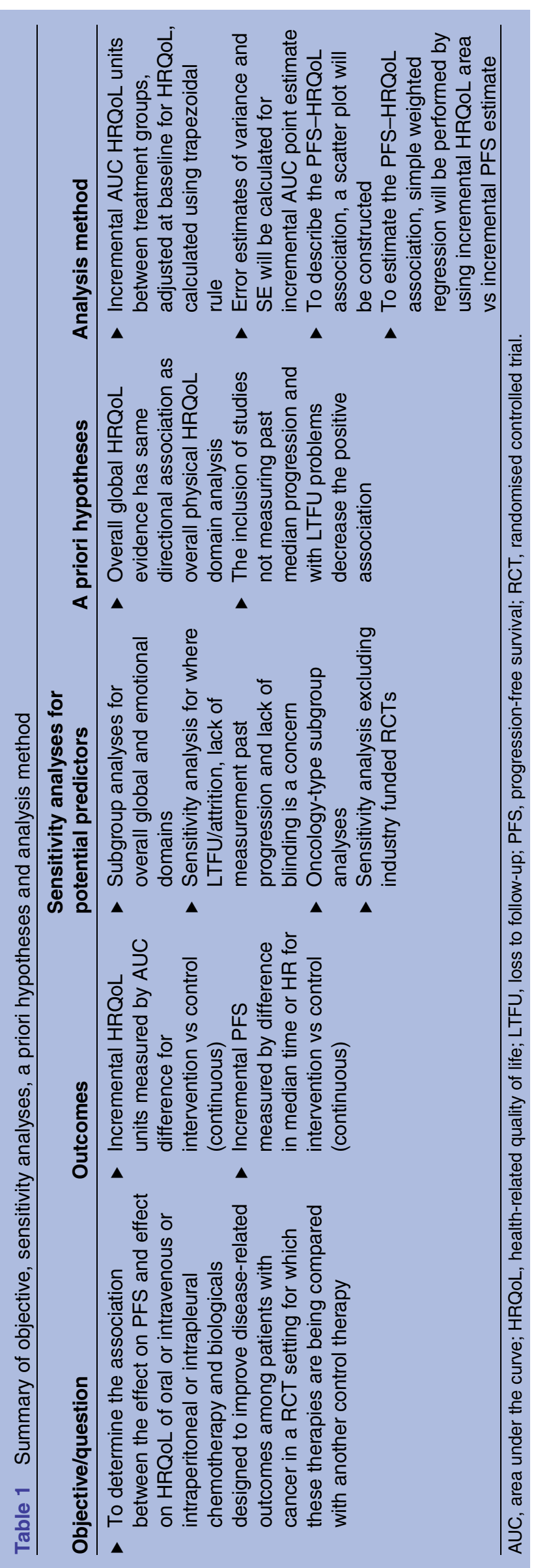


underestimate the PFS-HRQoL association. Since, to the extent that this problem occurs, we will be underestimating the PFS-HRQoL association, and this association can only increase in positive magnitude with longer HRQoL follow-up. Finding a strong positive HRQoLPFS association will be definitive. However, if in many studies we find a large difference in duration of follow-up in HRQoL and PFS, a finding of a weak or absent association will not be definitive.

Second, association does not necessarily imply causality, and with a plethora of unknown potential confounding factors linking PFS to HRQoL, there could be unknown underlying mechanisms having an effect on PFS and HRQoL. Additionally, we may not be able to distinguish between two explanations of an association between PFS and HRQoL: one in which the difference in tumour burden in those with and without recurrence directly results in difference in HRQoL, versus knowledge of having progression compared to being progression-free possibly reduces anxiety and increases HRQoL by that mechanism. It is possible that domain by domain analyses (for instance, if emotional function but not physical function improves, it might suggest the latter mechanism of effect) may help elucidate this issue. Also, we plan on including all HRQoL assessments, most of which will be prior to disease progression, in order to minimise the bias inherent in HRQoL collected on the day of documentation of progression.

Finally, although ideally we would stratify our HRQoL data by all known confounding factors, such as by type of progression (ie, radiographic vs clinical) or censoring due to death, we cannot perform these stratifications because of the nature of published HRQoL data. However, since many of these confounders are expected to be relatively well balanced between trial arms, we do not anticipate this being a major concern.

\section{Study implications}

Thus far, only one study has attempted to evaluate and provide systematic evidence on the PFS-HRQoL association. The AHRQ report conducted a qualitative analysis of a very limited amount of data, resulting in inconclusive results. Our more comprehensive search will result in a greater amount of retrieved data, which will allow for quantitative analyses providing stronger and more robust conclusions regarding the PFS-HRQoL association.

The PFS-HRQoL association is of primary concern to all stakeholders and decision makers, including patients, clinicians and payers. This is especially true given the ever increasing number of regulatory approved oncology drugs based on PFS benefit, even when no OS advantages are evident. This study will provide systematic research on a current evidence gap that exists in the oncology world. In order for PFS-based oncology treatments to conform to evidence-based medicine, systematic research on the PFSHRQoL association needs to be produced, so it can be integrated with individual clinical expertise in making decisions about the care of individual patients.
One area that this study will most likely challenge will be the conduct of oncology RCTs. Given the nature of our data, it is likely that one of the conclusions of this study will be that RCT authors need to design and run studies in a way as to minimise attrition and LTFU problems, as well as follow patients well past median progression, in order to, for the time being, measure what is most important to patients, HRQoL, and then only substitute PFS when its validity as a surrogate is definitively established.

The findings of this study may produce evidence that challenges conventional thinking regarding the surrogacy of PFS for HRQoL, or it may produce evidence that validates PFS as a surrogate for HRQoL. Regardless, it will contribute evidence that will support improved patient care, either through potentially changing research/clinical practices, guidelines and policies grounded in PFS or through providing knowledge that supports the current practices being employed.

\section{Author affiliations}

${ }^{1}$ Department of Clinical Epidemiology and Biostatistics, McMaster University, Hamilton, Ontario, Canada

${ }^{2}$ Faculty of Health Sciences, McMaster University, Hamilton, Ontario, Canada ${ }^{3}$ Department of Oncology, Queen's University, Kingston, Ontario, Canada ${ }^{4}$ Health Sciences Library, McMaster University, Hamilton, Ontario, Canada ${ }^{5}$ The Research Institute of St. Joseph's Healthcare, Hamilton, Ontario, Canada

Contributors BK and FX are the guarantors. BK drafted the manuscript. BK, GG, MB and FX developed the selection criteria. BK, GG and MB developed the data extraction criteria and risk of bias assessment strategy. BK and NB developed the search strategy. LT, FX and MB provided statistical expertise. LT and BK developed the statistical methodology. MB provided oncology-specific expertise. All authors read, provided feedback and approved the final manuscript.

Competing interests None declared.

Provenance and peer review Not commissioned; externally peer reviewed.

Open Access This is an Open Access article distributed in accordance with the Creative Commons Attribution Non Commercial (CC BY-NC 4.0) license, which permits others to distribute, remix, adapt, build upon this work noncommercially, and license their derivative works on different terms, provided the original work is properly cited and the use is non-commercial. See: http:// creativecommons.org/licenses/by-nc/4.0/

\section{REFERENCES}

1. Peppercorn JM, Smith TJ, Helft PR, et al. American society of clinical oncology statement: toward individualized care for patients with advanced cancer. J Clin Oncol 2011;29:755-60.

2. Booth CM, Eisenhauer EA. Progression-free survival: meaningful or simply measurable? J Clin Oncol 2012;30:1030-3.

3. Guyatt G, Montori V, Devereaux PJ, et al. Patients at the center: in our practice, and in our use of language. ACP J Club 2004;140: A11-12.

4. Pazdur R. Endpoints for assessing drug activity in clinical trials. Oncologist 2008;13(Suppl 2):19-21.

5. Fallowfield LJ, Fleissig A. The value of progression-free survival to patients with advanced-stage cancer. Nat Rev Clin Oncol 2012;9:41-7.

6. Gutman SI, Piper M, Grant MD, et al. Progression-free survival: what does it mean for psychological well-being or quality of life? Methods Research Report. (Prepared by the Blue Cross and Blue Shield Association Technology Evaluation Center Evidence-based Practice Center under Contract No. 290-2007-10058-I.) AHRQ Publication No. 13-EHC074-EF. Rockville, MD: Agency for Healthcare Research and Quality. April 2013. www.effectivehealthcare.ahrq.gov/reports/ final.cfm.

7. Higgins JPT, Green S, eds. Cochrane handbook for systematic reviews of interventions. The Cochrane Collaboration, 2011. 
8. Moher D, Shamseer L, Clarke M, et al. Preferred reporting items for systematic review and meta-analysis protocols (PRISMA-P) 2015 statement. Syst Rev 2015;4:1.

9. Shamseer L, Moher D, Clarke M, et al. Preferred reporting items for systematic review and meta-analysis protocols (PRISMA-P) 2015: elaboration and explanation. BMJ 2015;349:g7647.

10. Booth A, Clarke M, Dooley G, et al. The nuts and bolts of PROSPERO: an international prospective register of systematic reviews. Syst Rev 2012;1:2.

11. Booth $A$, Clarke $M$, Ghersi $D$, et al. An international registry of systematic-review protocols. Lancet 2011;377: $108-9$.

12. Abridged Index Medicus (AIM or "Core Clinical") Journal Titles. 09 July 2003 ed: U.S. National Library of Medicine. https://www.nlm.nih. gov/bsd/aim.html
13. SEER Training Modules, Review: Categories of Cancer: US. National Institutes of Health, National Cancer Institute. http://training.seer.cancer.gov/disease/categories/review.html

14. Types of cancer: Cancer Research UK. (updated 28 Oct 2014). http://www.cancerresearchuk.org/about-cancer/what-is-cancer/ how-cancer-starts/types-of-cancer\#main

15. NCl Dictionary of Cancer Terms-Chemotherapy: National Cancer Institute. http://www.cancer.gov/publications/dictionaries/cancerterms? cdrid $=45214$

16. NCl Dictionary of Cancer Terms-Biological Therapy: National Cancer Institute. http://www.cancer.gov/publications/dictionaries/ cancer-terms? cdrid $=45617$

17. Chapter 8: assessing risk of bias in included studies. The Cochrane Collaboration, 2011. (updated Mar 2011). http://www. cochrane-handbook.org 